\title{
VERY LONG BASELINE ARRAY IMAGING OF PARSEC-SCALE RADIO EMISSIONS IN NEARBY RADIO-QUIET NARROW-LINE SEYFERT 1 GALAXIES
}

\author{
Akihiro Doi ${ }^{1,2}$, Keitchi Asada ${ }^{3}$, Kenta Fujisawa $^{4}$, Hiroshi Nagai $^{5}$, Yoshiaki Hagiwara ${ }^{5}$, \\ KIYOAKI WAJIMA ${ }^{6}$, AND MAKOTO INOUE ${ }^{3}$ \\ ${ }^{1}$ The Institute of Space and Astronautical Science, Japan Aerospace Exploration Agency, 3-1-1 Yoshinodai, Chuou-ku, Sagamihara, \\ Kanagawa 252-5210, Japan; akihiro.doi@vsop.isas.jaxa.jp \\ ${ }^{2}$ Department of Space and Astronautical Science, The Graduate University for Advanced Studies, 3-1-1 Yoshinodai, \\ Chuou-ku, Sagamihara, Kanagawa 252-5210, Japan \\ ${ }^{3}$ Academia Sinica Institute of Astronomy and Astrophysics, P.O. Box 23-141, Taipei 10617, Taiwan \\ ${ }^{4}$ The Research Institute of Time Studies, Yamaguchi University, 1677-1 Yoshida, Yamaguchi, Yamaguchi 753-8511, Japan \\ ${ }^{5}$ National Astronomical Observatory, 2-21-1 Osawa, Mitaka, Tokyo 181-8588, Japan \\ ${ }^{6}$ Shanghai Astronomical Observatory, Chinese Academy of Sciences, Shanghai 200030, China \\ Received 2012 August 29; accepted 2013 January 14; published 2013 February 15
}

\begin{abstract}
We conducted Very Long Baseline Array (VLBA) observations of seven nearby narrow-line Seyfert 1 (NLS1) galaxies at $1.7 \mathrm{GHz}(\lambda 18 \mathrm{~cm})$ with milliarcsecond resolution. This is the first systematic very long baseline interferometry study focusing on the central parsec-scale regions of radio-quiet NLS1s. Five of the seven were detected at a brightness temperature of $\gtrsim 5 \times 10^{6} \mathrm{~K}$ and contain radio cores with high brightness temperatures of $>6 \times 10^{7} \mathrm{~K}$, indicating a nonthermal process driven by jet-producing central engines as in radio-loud NLS1s and other active galactic nucleus classes. VLBA images of MRK 1239, MRK 705, and MRK 766 exhibit parsec-scale jets with clear linear structures. A large portion of the radio power comes from diffuse emission components that are distributed within the nuclear regions $(\lesssim 300 \mathrm{pc})$, which is a common characteristic throughout the observed NLS1s. Jet kinetic powers limited by the Eddington limit may be insufficient to allow the jets to escape to kiloparsec scales for these radio-quiet NLS1s with low-mass black holes of $\lesssim 10^{7} M_{\odot}$.
\end{abstract}

Key words: galaxies: active - galaxies: individual (MRK 110, MRK 705, MRK 1239, NGC 4051, MRK 766

(NGC 4253), MRK 783, AKN 564) - galaxies: jets - galaxies: Seyfert - radio continuum: galaxies

\section{INTRODUCTION}

Narrow-line Seyfert 1 galaxies (NLS1s) are identified by their optical properties: a flux ratio $[\mathrm{O} \mathrm{III}] / \mathrm{H} \beta<3$, and Balmer lines only slightly broader than forbidden lines (Osterbrock \& Pogge 1985 ), defined as FWHM $(\mathrm{H} \beta)<2000 \mathrm{~km} \mathrm{~s}^{-1}$ (Goodrich 1989). NLS1s make up $\sim 20 \%$ of an optically selected active galactic nucleus (AGN) sample from the Sloan Digital Sky Survey (SDSS; e.g., Zhou et al. 2006). Recently, $\gamma$-ray emissions have been detected from several very radio-loud NLS1s by the Large Area Telescope on board the Fermi Gamma-ray Space Telescope (Abdo et al. 2009a, 2009b; Foschini 2011); it has been argued that these NLS1s may represent a third class of $\gamma$-ray emitting AGNs along with blazars and radio galaxies. The detected $\gamma$-rays strongly suggest the presence of blazar-like phenomena as a consequence of relativistic beaming effects due to a fast jet aligned close to our line of sight (Blandford \& Konigl 1979). Moreover, the presence of highly relativistic jets in radio-loud NLS1s has been implied on the basis of radio flux variability observations (Zhou et al. 2003; Doi et al. 2006, 2011; Yuan et al. 2008), very-long baseline interferometry (VLBI) imaging (Doi et al. 2006, 2011; Giroletti \& Panessa 2009; D’ Ammando et al. 2012), and modeling of the spectral energy distributions (Zhou et al. 2007; Yuan et al. 2008; Abdo et al. 2009b). Kiloparsec $(\mathrm{kpc})$-scale radio emissions are associated with several radioloud NLS1s, including two $\gamma$-ray emitting NLS1s (Antón et al. 2008; Gliozzi et al. 2010; Doi et al. 2012). However, these powerful jet phenomena are observed in only a small part of the NLS1 populations. In fact, the fraction of radio-loud objects ${ }^{7}$ in

\footnotetext{
The radio loudness $R$ is defined as the ratio of the $5 \mathrm{GHz}$ radio flux density and optical $B$-band flux density. A threshold of $R=10$ separating radio-loud and radio-quiet objects (Kellermann et al. 1989) is frequently used.
}

the NLS1 population is significantly low (Zhou \& Wang 2002; Stepanian et al. 2003; Greene et al. 2006; Zhou et al. 2006; Yuan et al. 2008), $\sim 7 \%$ with $R>10$ and $\sim 2.5 \%$ with $R>100$ (Komossa et al. 2006), compared with normal Seyfert galaxies and quasars ( 10\%-15\%; e.g., Ivezić et al. 2002). Similarly, kpc-scale radio emissions are less common in NLS1s than in broad-line AGNs (Doi et al. 2012). The radio jet characteristics of a huge amount of the radio-quiet NLS1 population behind these extremes are little known.

NLS1s as a class are considered to have weak jet activities. A radio-imaging study at arcsecond resolution using the Very Large Array (VLA) revealed that nearby (radio-quiet) NLS1s, in most cases, are unresolved radio sources $[\lesssim 300$ parsec (pc)] with modest radio luminosities $\left(10^{20}-10^{23} \mathrm{~W} \mathrm{~Hz}^{-1}\right)$ and steep radio spectra $\left(\alpha<-0.5\right.$, where $S_{v} \propto \nu^{\alpha}$, and $S_{v}$ is the flux density at the frequency $v$ ). Thus, NLS1s are not remarkably different from normal Seyfert galaxies at radio wavelengths (Ulvestad et al. 1995; see also Moran 2000; Zuther et al. 2011), although they exhibit many distinctive optical-X-ray properties. In addition to the unusually narrow Balmer lines, NLS1s exhibit several extreme properties such as strong permitted $\mathrm{Fe}$ II emission lines (Boroson \& Green 1992), rapid X-ray variability (Pounds et al. 1995; Leighly 1999b), steep soft X-ray spectra (Wang et al. 1996; Boller et al. 1996; Leighly 1999a), and frequently observed blueshifted line profiles (Zamanov et al. 2002; Boroson 2005; Leighly \& Moore 2004). It has been suggested that these properties are related to high-mass accretion rates close to the Eddington limit (Boroson \& Green 1992; Brandt \& Boller 1998; Sulentic et al. 2000; Mineshige et al. 2000) on relatively low-mass black holes $\left(\sim 10^{5}-10^{7.5} M_{\odot}\right.$; Peterson et al. 2000; Hayashida 2000; Grupe \& Mathur 2004; Zhou et al. 2006). A high accretion rate on a low-mass black hole supports the radio quiescence of the NLS1 class, according to many studies 
using large AGN samples: a positive correlation between radio loudness and the black hole mass (Laor 2000; Lacy et al. 2001; Dunlop et al. 2003; McLure \& Jarvis 2004; Metcalf \& Magliocchetti 2006) and an anti-correlation between radio loudness and the accretion rate (Ho 2002; Lacy et al. 2001; Maccarone et al. 2003; Greene et al. 2006). Similarly, Merloni et al. (2003) discovered "a fundamental plane of black hole activity" in three-dimensional space consisting of the radio luminosity, X-ray luminosity, and black hole mass (ranging from $10^{1}$ to $10^{10} M_{\odot}$ ) for samples of X-ray binaries, our Galactic center, low-luminosity AGNs, Seyfert galaxies, and quasars. These tendencies in jet activity can be attributed to nonlinear dependences on the black hole mass and accretion rate (Heinz $\&$ Sunyaev 2003). Thus, the weak radio emissions of NLS1s as a class can be understood in the framework established for other AGN classes.

However, the picture of a nonthermal jet origin for the observed radio emissions of NLS1s has not been fully established yet, particularly for the radio-quiet subclass, because of the difficulty of VLBI observations of weak radio sources. Previously, a nonthermal jet origin for radio-quiet quasars was established by a series of VLBI detections (Blundell \& Beasley 1998; Ulvestad et al. 2005). The first VLBI study focusing on the NLS1 class at milliarcsecond (mas) resolutions examined the very radioloud narrow-line quasar PMN J0948+0022 using the Very Long Baseline Array (VLBA; Doi et al. 2006). A Doppler-beaming effect of highly relativistic jets is required to explain the observed extremely high brightness and rapid flux variability in the VLBA images. Subsequent $\gamma$-ray detection by Fermi/LAT followed (Abdo et al. 2009a). Doi et al. (2007) detected five additional radio-loud NLS1s using the Japanese VLBI Network and established the picture of a nonthermal process in the radio-loud NLS1 subclass. Gu \& Chen (2010) reported compact radio structures for three of the five radio-loud NLS1s using VLBA imaging. Doi et al. (2011) found evidence of pc-scale jet structures in all five radio-loud NLS1s and pointed out (1) the presence of relativistic nonthermal jets, which are essentially the same as those of other radio-loud AGN classes; and (2) the existence of both apparently radio-loud NLS1s owing to Doppler beaming and intrinsically radio-loud NLS1s. Several $\gamma$-ray emitting NLS1s have been observed in survey programs of VLBI radio sources-SBS 0846+513 (Kovalev et al. 2007; D'Ammando et al. 2012), 1H 0323+342 (Zhou et al. 2007), and PKS 1502+036 (Fey \& Charlot 2000; see also Dallacasa et al. 1998 for a study of this object as a compact radio galaxy candidate) — and are now being monitored by VLBA in the MOJAVE program. On the other hand, there have been only a handful of images of radio-quiet NLS1s: MRK 766, AKN 564 (Lal et al. 2004), NGC 5506 (Middelberg et al. 2004), NGC 4051 (Giroletti \& Panessa 2009), and MRK 1239 (Orienti \& Prieto 2010) as part of a study of Seyfert galaxies (not focused on NLS1s). A systematic VLBI study of radio-quiet NLS1s is crucial for understanding the nature of their radio-quiescent central engines with the lowest-mass black holes at the highest accretion rates in AGN classes. Furthermore, radio-loud NLS1s should be discussed as extreme examples on the basis of more solid knowledge of radio-quiet objects that are the majority in the NLS1 population.

In the present paper, we report VLBI imaging of seven nearby radio-quiet NLS1s. In Section 2, we describe the sample selection. In Section 3, we describe the observations and

\footnotetext{
8 Monitoring of jets in AGNs with VLBA experiments (Lister et al. 2009).
}

imaging processes. The observational results are presented in Section 4 . We discuss the observed radio properties of the radioquiet NLS1s in Section 5. Finally, we summarize our studies in Section 6. The observed properties of individual radio-quiet NLS1s are described in the Appendix. Throughout this paper, a $\Lambda \mathrm{CDM}$ cosmology with $H_{0}=71 \mathrm{~km} \mathrm{~s}^{-1} \mathrm{Mpc}^{-1}, \Omega_{\mathrm{M}}=0.27$, and $\Omega_{\Lambda}=0.73$ is adopted (Komatsu et al. 2009).

\section{SAMPLE}

A nearby radio-quiet NLS1 sample consisting of seven objects was made for this VLBI imaging study (Table 1). We selected from the 205 NLS1s listed in "Quasars and Active Galactic Nuclei," 10th Ed. (Véron-Cetty \& Véron 2001), and the 64 NLS1s in "Spectrophotometric Atlas of NLS1s" (VéronCetty et al. 2001). We processed the following selection criteria step by step: (1) redshift $z<0.1$ (129 sources survived), (2) inclusion in the VLA Faint Images of the Radio Sky at TwentyCentimeters (FIRST; Becker et al. 1995), 03Apr11 version (60 sources survived), (3) existence of a radio counterpart with an offset of $<1^{\prime \prime}$ in the FIRST image (33 sources survived), (4) a peak intensity of more than $5 \mathrm{mJy}^{\text {beam }}{ }^{-1}$ (13 sources survived), and (5) availability of a radio position measured at a resolution of $<0$ '. 5 from literature or archival data (to enable processing by VLBA correlator as described in Section 3.1 in detail; 7 sources survived). One of the selected NLS1s, MRK 1388, has sometimes been classified as a type 1.9 or 2 Seyfert (e.g., Goodrich 1989) with an unresolvable broadline component (Osterbrock 1985; Veron et al. 1997); hence, we excluded it from our sample. In addition, we included AKN 564 , one of the best-studied NLS1s, although it does not properly satisfy criterion (2); we substituted VLA archival data (AT149) obtained at $\sim 5$ arcsec resolution as equivalent to the FIRST data. As a result, seven sources were selected. They are genuine NLS1s according to the optical definitions and also other aspects such as the Fe II line strength, soft X-ray spectrum, and black hole mass (Columns 3-9 in Table 1). All seven NLS1s are radio quiet by the definition of radio loudness using the nuclear optical and total radio flux densities ( $R_{*}$; Column 11 in Table 1 ). They all, except for MRK 110, are compact radio sources covering $\lesssim 300$ pc in VLA images (Column 12 in Table 1 ).

\section{OBSERVATIONS AND DATA REDUCTION}

\subsection{Sky Positions}

The field of view (FOV) in this study is limited for statistical reasons. Searching for emission with a detection limit of $4 \sigma$ requires an FOV limited to $\sim 32,000$ points, corresponding to a radius of $\sim 100$ times the synthesized beam on the sky. The expected size of the synthesized beam is about 11 mas $\times$ 5 mas aligned nearly north-south at the declinations of our sample: the $\mathrm{FOV}^{9}$ is supposed to be $\sim 1^{\prime \prime} .1 \times 0^{\prime \prime} .5$. Hence, we attempted to determine the positions for correlation (the phasetracking centers) to sub-arcsec accuracy during sample selection (Section 2).

For each NLS1, we obtained the radio position with an uncertainty of $\sim 0$.' 1 in images from the VLA A-array configuration in the archive (AM384, AK394, AB973, and AL410) and/or in the literature (Ulvestad \& Wilson 1984; Ulvestad et al. 1995; Kukula et al. 1998; Nagar et al. 1999; Thean et al. 2000; Schmitt

\footnotetext{
9 The bandwidth smearing for a nominal continuum mode and the time-average smearing for $2 \mathrm{~s}$ integration in the VLBA correlator restrict the FOV to $\sim 7^{\prime \prime}$ and $\sim 15^{\prime \prime}$, respectively (Thompson et al. 1986; Wrobel 1995).
} 
Table 1

Nearby Radio-quiet NLS1 Sample for VLBA Observations

\begin{tabular}{|c|c|c|c|c|c|c|c|c|c|c|c|c|}
\hline Name & (2) & $\begin{array}{c}\text { FWHM }(\mathrm{H} \beta) \\
\left(\mathrm{km} \mathrm{s}^{-1}\right) \\
(3)\end{array}$ & $\begin{array}{c}{\left[\mathrm{O}_{\mathrm{III}}\right] / \mathrm{H} \beta} \\
\text { (4) }\end{array}$ & $\begin{array}{c}R_{4570} \\
(5)\end{array}$ & $\begin{array}{l}\text { Ref. } \\
(6)\end{array}$ & $\begin{array}{l}\Gamma \\
\text { (7) }\end{array}$ & $\begin{array}{c}\log M_{\mathrm{BH}} \\
\left(M_{\odot}\right) \\
(8)\end{array}$ & $\begin{array}{l}\text { Ref. } \\
(9)\end{array}$ & $\begin{array}{c}S_{1.4 \mathrm{GHz}}^{\mathrm{total}} \\
(\mathrm{mJy}) \\
(10)\end{array}$ & $\begin{array}{l}R_{*} \\
(11)\end{array}$ & $\begin{array}{c}\Theta \\
(\mathrm{pc}) \\
(12)\end{array}$ & Ref. \\
\hline MRK 110 & 0.0353 & 1760 & 0.71 & 0.26 & 1 & 2.59 & 6.75 & 4 & 10.1 & 1.8 & 2100 & 8 \\
\hline MRK 705 & 0.0292 & 1790 & 0.41 & 0.36 & 2 & 2.53 & 6.72 & 5 & $8.5^{\mathrm{c}}$ & 2.1 & $<55$ & 9 \\
\hline MRK 1239 & 0.0199 & 1075 & 1.29 & 0.63 & 2 & 4.06 & 5.89 & 5 & 62.8 & 0.69 & 46 & 10 \\
\hline NGC 4051 & 0.0023 & 1120 & 0.55 & 0.25 & 2 & 3.04 & 6.13 & 4 & $14.7^{\mathrm{d}}$ & 0.62 & 330 & 11 \\
\hline MRK 766 & 0.0129 & 1630 & 0.35 & 1.83 & 2 & 2.63 & 6.80 & 6 & 39.7 & 0.63 & 65 & 12 \\
\hline MRK 783 & 0.0672 & 1655 & 2.29 & 0.49 & 2,3 & $1.30^{\mathrm{a}}$ & 7.16 & 7 & 33.2 & 2.0 & $<380$ & 13 \\
\hline AKN 564 & 0.0247 & 865 & 0.92 & 0.67 & 2 & $3.4^{\mathrm{b}}$ & 6.75 & 4 & 29.1 & 0.44 & 320 & 9 \\
\hline
\end{tabular}

Notes. Column 1: source name; Column 2: redshift; Column 3: FWHM of H $\beta$ line width of broad-line component; Column 4: flux ratio of [O III] $\lambda 5007$ to H $\beta$; Column 5: flux ratio of Fe II multiplets in the range 4434-4684 $\AA$ to $\mathrm{H} \beta$; Column 6: reference for optical-line emissions; Column 7: ROSAT soft X-ray photon index at 0.1-2.4 keV (Pfefferkorn et al. 2001); Column 8: black hole mass; Column 9: reference for the black hole mass; Column 10: NRAO VLA Sky Survey (NVSS; 45") flux density at $1.4 \mathrm{GHz}$ as total flux density; Column 11: radio loudness defined as the ratio of $k$-corrected $5 \mathrm{GHz}$ radio to optical $B$-band flux densities (Kellermann et al. 1989). We calculated the radio loudness using the NVSS flux density and SDSS 7DR PSF magnitude (Abazajian et al. 2009) extinction-corrected by assuming a Balmer decrement of $\mathrm{H} \alpha / \mathrm{H} \beta=3.1$ for all sources, except AKN 564, for which we used a $V$-band nuclear magnitude (Mullaney \& Ward 2008). We assume $\alpha=-0.7$ for radio (except for MRK 1239, MRK 766, and AKN 564; see the Appendix) and $\alpha=-0.61$ for optical (Grupe et al. 2010) for the $k$ corrections. Column 12: maximum extent of radio structure from nucleus in VLA images at arcsecond resolution; Column 13: reference for the radio size.

${ }^{a}$ A large uncertainty $( \pm 0.72)$.

b Boller et al. 1996.

c Flux density from FIRST $\left(\sim 5^{\prime \prime}\right)$ at $1.4 \mathrm{GHz}$ to avoid the contamination from an unrelated source $\sim 30^{\prime \prime}$ south (Gallo et al. 2005).

${ }^{\mathrm{d}}$ Flux density from VLA A-configuration $\left(\sim 1^{\prime \prime} .2\right)$ at $1.4 \mathrm{GHz}$ (Ho \& Ulvestad 2001), because the NVSS emission is contaminated by the contribution of a host galaxy (Baum et al. 1993).

References. (1) Grupe et al. 2004; (2) Véron-Cetty et al. 2001; (3) Kovačević et al. 2010; (4) Kaspi et al. 2000; (5) Ryan et al. 2007; (6) Done \& Gierliński 2005; (7) Wang \& Lu 2001; (8) Kukula et al. 1998; (9) Schmitt et al. 2001; (10) this paper (the Appendix); (11) Ho \& Ulvestad 2001; (12) Ulvestad et al. 1995; (13) Ulvestad \& Wilson 1984.

Table 2

Parameters of Phase-reference VLBA Observations

\begin{tabular}{|c|c|c|c|c|c|c|c|}
\hline \multirow{2}{*}{$\begin{array}{l}\text { Name } \\
\text { (1) }\end{array}$} & \multicolumn{2}{|c|}{ Phase-tracking Center (J2000) } & \multirow{2}{*}{$\begin{array}{l}\text { Ref. } \\
\text { (4) }\end{array}$} & \multicolumn{2}{|c|}{ Astrometric Position (J2000.0) } & \multirow{2}{*}{$\begin{array}{c}\text { Reference } \\
\text { Source } \\
(7)\end{array}$} & \multirow{2}{*}{$\begin{array}{l}\Delta \theta \\
\left({ }^{\circ}\right) \\
(8)\end{array}$} \\
\hline & $\begin{array}{c}\text { R.A. (h m s} \text { ) } \\
\text { (2) }\end{array}$ & $\begin{array}{c}\operatorname{Decl} .\left({ }^{\circ \prime \prime \prime}\right) \\
(3)\end{array}$ & & $\begin{array}{c}\text { R.A. (h m s} \text { ) } \\
\text { (5) }\end{array}$ & $\begin{array}{c}\operatorname{Decl} .\left({ }^{\circ} \prime \prime \prime\right. \\
(6)\end{array}$ & & \\
\hline MRK 110 & 092512.8500 & +521710.388 & 1 & 092512.8476 & +521710.387 & J0932+5306 & 1.40 \\
\hline MRK 705 & 092603.2763 & +124403.659 & 2 & $\ldots{ }^{\mathrm{a}}$ & $\ldots$ & $\mathrm{J} 0931+1414$ & 1.94 \\
\hline MRK 1239 & 095219.0996 & -013643.553 & 3 & 095219.0980 & -013643.546 & $\mathrm{~J} 0948+0022$ & 2.16 \\
\hline NGC 4051 & 120309.6105 & +443152.680 & 4 & $\ldots{ }^{b}$ & $\ldots$ & $\mathrm{J} 1155+4555$ & 1.98 \\
\hline MRK 766 & 121826.5186 & +294846.471 & 5 & $\ldots^{\mathrm{a}}$ & $\ldots$ & $\mathrm{J} 1217+3007$ & 0.33 \\
\hline MRK 783 & 130258.8430 & +162427.748 & 6 & 130258.8438 & +162427.777 & $\mathrm{~J} 1311+1658$ & 2.09 \\
\hline AKN 564 & 224239.3397 & +294330.915 & 2 & $\ldots{ }^{b}$ & $\ldots$ & $\mathrm{J} 2236+2828$ & 1.85 \\
\hline
\end{tabular}

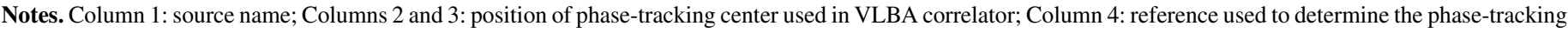

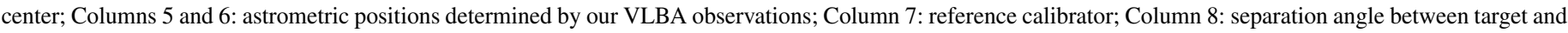
reference calibrator.

${ }^{a}$ Position is not available because of detection in self-calibrated image.

b Non-detection.

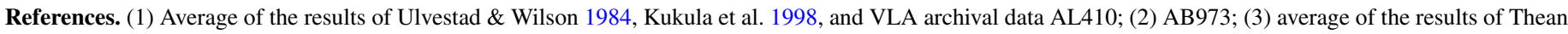

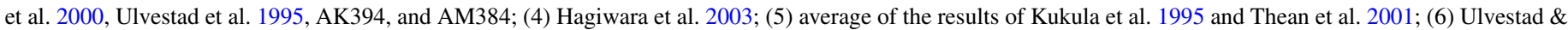
Wilson 1984.

et al. 2001). We used the National Radio Astronomy Observatory (NRAO) SCHED software when it was necessary to convert a B1950 VLA position to J2000. The phase-tracking centers used in our VLBI observations are listed in Table 2.

\subsection{Observations}

We observed the seven NLS1s on 2005 May 9 at $1.7 \mathrm{GHz}$ using 10 antennas of the VLBA at the NRAO (observation code BD106). A left circular polarization was received at a center frequency of $1.667 \mathrm{GHz}$ with a total bandwidth of $32 \mathrm{MHz}$. We observed in the phase-referencing mode, which allows us to derive calibration parameters for instrumental and atmospheric effects from the observations of nearby strong compact radio sources ("calibrators;" Beasley \& Conway 1995). The phasereference cycle periods were 5 minutes, and the total on-source time was about 40 minutes for each target.

\subsection{Data Reduction}

Standard calibration procedures for VLBA phase referencing were applied during data reduction using the Astronomical Image Processing System (AIPS; Greisen 2003). Inappropriate Earth-orientation parameters were corrected using the AIPS task CLCOR. Amplitude calibration using a priori gain values together with system temperatures measured during the observations was applied; the accuracy is typically $5 \%$. The ionospheric dispersive delay was corrected using a global ionospheric model and the 
Table 3

Results of VLBA Observations

\begin{tabular}{|c|c|c|c|c|c|c|c|c|c|c|c|}
\hline Name & $\begin{array}{c}\begin{array}{c}\text { VLBIcore } \\
1.7 \mathrm{GHz}\end{array} \\
\left(\mathrm{mJy} \mathrm{beam}^{-1}\right) \\
(2)\end{array}$ & $\begin{array}{c}S_{1.7 \mathrm{GHz}}^{\mathrm{VLBIcore}} \\
(\mathrm{mJy}) \\
(3)\end{array}$ & $\begin{array}{c}S_{1.7 \mathrm{GHz}}^{\text {VLBitotal }} \\
(\mathrm{mJy}) \\
(4)\end{array}$ & $\begin{array}{c}\sigma \\
\left(\mathrm{mJy} \mathrm{beam}^{-1}\right) \\
(5)\end{array}$ & $\begin{array}{c}\theta_{\mathrm{maj}} \\
(\mathrm{mas}) \\
(6)\end{array}$ & $\begin{array}{c}\theta_{\min } \\
(\mathrm{mas}) \\
(7)\end{array}$ & $\begin{array}{l}\text { P.A. } \\
\left({ }^{\circ}\right) \\
(8)\end{array}$ & $\begin{array}{c}T_{\mathrm{B}} \\
\left(\times 10^{7} \mathrm{~K}\right) \\
(9)\end{array}$ & $\begin{array}{c}\theta_{10 \mathrm{pc}} \\
(\mathrm{mas}) \\
(10)\end{array}$ & $\begin{array}{c}S_{1.7 \mathrm{GHz}}^{<10 \mathrm{pc}} \\
(\mathrm{mJy}) \\
(11)\end{array}$ & $\begin{array}{c}f_{1.7 \mathrm{GHz}}^{10 \mathrm{pc}} \\
(12)\end{array}$ \\
\hline MRK 110 & $1.1 \pm 0.1$ & $1.2 \pm 0.2$ & 1.1 & 0.128 & 10.9 & 4.4 & -12.7 & $>6.5$ & 14.4 & 1.1 & 0.13 \\
\hline MRK 705 & $1.8 \pm 0.2$ & $1.8 \pm 0.3$ & 3.3 & 0.144 & 11.2 & 4.9 & -0.8 & $>8.7$ & 17.3 & 1.8 & 0.24 \\
\hline MRK 1239 & $2.4 \pm 0.2$ & $4.8 \pm 0.5$ & 20.0 & 0.169 & 11.3 & 4.5 & -2.5 & 7.4 & 25.1 & 13.1 & 0.23 \\
\hline NGC 4051 & $<0.7$ & $<0.7$ & $<0.7$ & 0.143 & 11.2 & 4.3 & $\ldots$ & $<1.0$ & 121 & $<0.7$ & $<0.05$ \\
\hline MRK 766 & $1.3 \pm 0.1$ & $1.6 \pm 0.2$ & 2.5 & 0.103 & 11.2 & 4.4 & -4.1 & 8.4 & 38.4 & 2.4 & 0.07 \\
\hline MRK 783 & $1.4 \pm 0.1$ & $1.3 \pm 0.2$ & 1.3 & 0.129 & 10.8 & 4.4 & -6.7 & $>7.7$ & 7.9 & 1.3 & 0.04 \\
\hline AKN 564 & $<1.0$ & $<1.0$ & $<1.0$ & 0.208 & 11.8 & 4.5 & $\ldots$ & $<1.3$ & 20.4 & $<1.0$ & $<0.04$ \\
\hline
\end{tabular}

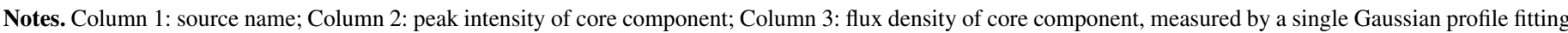

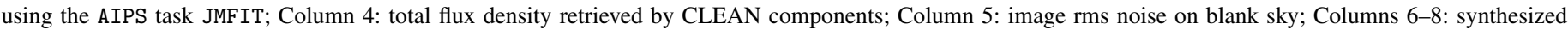

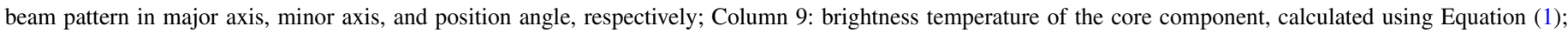

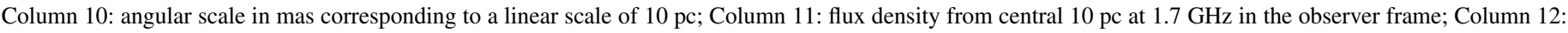

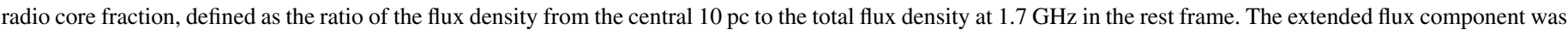

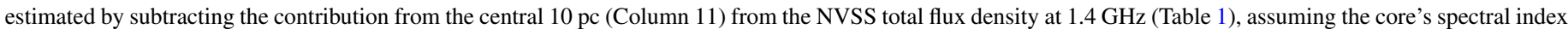
available in the literature or $\alpha=-0.5$ for values unavailable in the literature and $\alpha=-0.7$ for the extended component. See the Appendix for individual objects.

task TECOR. The time and spectral domains were carefully inspected, and data flagging was performed; there was no significant radio interference in the data.

Conservatively, we first searched for emission components with peak intensities of $>5 \sigma$ of the noise on images with natural weighting in an FOV of $1^{\prime \prime} .1 \times 0.5$, and also greater than $6 \sigma$ in an FOV of 3.' $0 \times 1$ 1".3 (Section 3.1). At this stage, we detected three sources: MRK 110, MRK 1239, and MRK 783. They initially appeared at positions offset by less than several tens of mas from the phase-tracking centers. After correcting the map centers to the emission peaks, we deconvolved the images using the task IMAGR (CLEAN) and measured the astrometric positions of the emissions using the task JMFIT (Table 2). The images of negative detection sources MRK 705, MRK 766, and AKN 564 showed quadrangular patterns resembling beam patterns. There were several emission candidates with intensities of $4 \sigma-5 \sigma$ in the images. The patterns seemed to contribute to increasing the image noise to levels significantly higher than the expected thermal noises. This suggested that significant emission does exist, but its power could be scattered owing to imperfect calibration of the phase-referenced array. The quality of the phase referencing is usually more or less degraded by the uncertainty in the correlator models, mainly regarding the residual atmospheric (troposphere and ionosphere) delay for each antenna's site. Hence, we attempted self-calibration in phase with a signal-to-noise ratio threshold of 2.5 using the task CALIB. Astrometric information was lost in the self-calibration procedure. We defined the success of self-calibration according to whether the solutions were found as a smooth-phase gradient for all the antennas through more than half the observing time on a target. The self-calibration was successful and emissions were detected for MRK 705, MRK 1239, MRK 766, and MRK 783. We smoothed and interpolated the solutions. The deconvolution and self-calibration algorithms were interactively applied a few times. As a result, NGC 4051 and AKN 564 were not detected with a detection limit of five times the image noise.

We used the Difmap software (Shepherd 1997) to make final images using the CLEAN algorithm from the calibrated visibilities (additional self-calibration was not performed in Difmap). We used step-by-step uniform, natural, and $(u, v)$ tapered weighting functions for CLEAN to retrieve both compact and diffuse components as much as possible. Consequently, we obtained self-calibrated images for four targets (MRK 705,
MRK 1239, MRK 766, and MRK 783) and a direct image for one target (MRK 110).

\section{RESULTS}

Five of the seven radio-quiet NLS1s were detected in the VLBA observations. The results of the image analyses are shown in Table 3. The images with natural weighting for the detected sources are shown in Figure 1. We also show images convolved with a restored beam size of $10 \mathrm{pc}$ in Figure 2. We describe the details for individual radio-quiet NLS1s in the Appendix.

\subsection{Brightness Temperatures}

The images of the five detected radio-quiet NLS1s show a compact component (core) with high brightness temperatures of $T_{\mathrm{B}}>6 \times 10^{7} \mathrm{~K}$ (Table 3 ), which were calculated from

$$
T_{\mathrm{B}}=1.8 \times 10^{9}(1+z) \frac{S_{v}}{v^{2} \phi_{\mathrm{maj}} \phi_{\min }}
$$

in kelvin at the rest frame, where $z$ is the redshift, $S_{v}$ is the flux density in mJy at the observing frequency $v$ in $\mathrm{GHz}$, and $\phi_{\text {maj }}$ and $\phi_{\min }$ in mas are the fitted FWHM of the major and minor axes of the component, respectively (cf. Ulvestad et al. 2005). If a source was unresolved, we adopted half the beam sizes as the upper limits to the source sizes $(\phi<\theta / 2)$.

The typical image noise of $\sim 0.41 \mathrm{mJy}^{\text {beam }}{ }^{-1}$ corresponds to $T_{\mathrm{B}} \sim 1.7 \times 10^{6} \mathrm{~K}$. Thus, VLBI imaging works as a highbrightness filter. Even diffuse emissions of $\sim 3 \sigma$ (the lowest contour level in the images) imply $T_{\mathrm{B}} \approx 5 \times 10^{6} \mathrm{~K}$, which is too high to attribute to any stellar origin. We conclude that all the detected VLBA emissions from these radio-quiet NLS1s are relevant to the activity of AGNs.

\subsection{Source Structures}

The five detected sources have compact emissions with high brightness temperatures. Furthermore, we can recognize significant structures in the images of MRK 1239 (Figure 1), MRK 705, and MRK 766 (Figure 2). A linear structure extending to either side of a brightness peak was clearly seen in MRK 1239. The visible radio extent was $\sim 100$ mas (corresponding to $\sim 40 \mathrm{pc}$ ), which was consistent with a slight elongation 

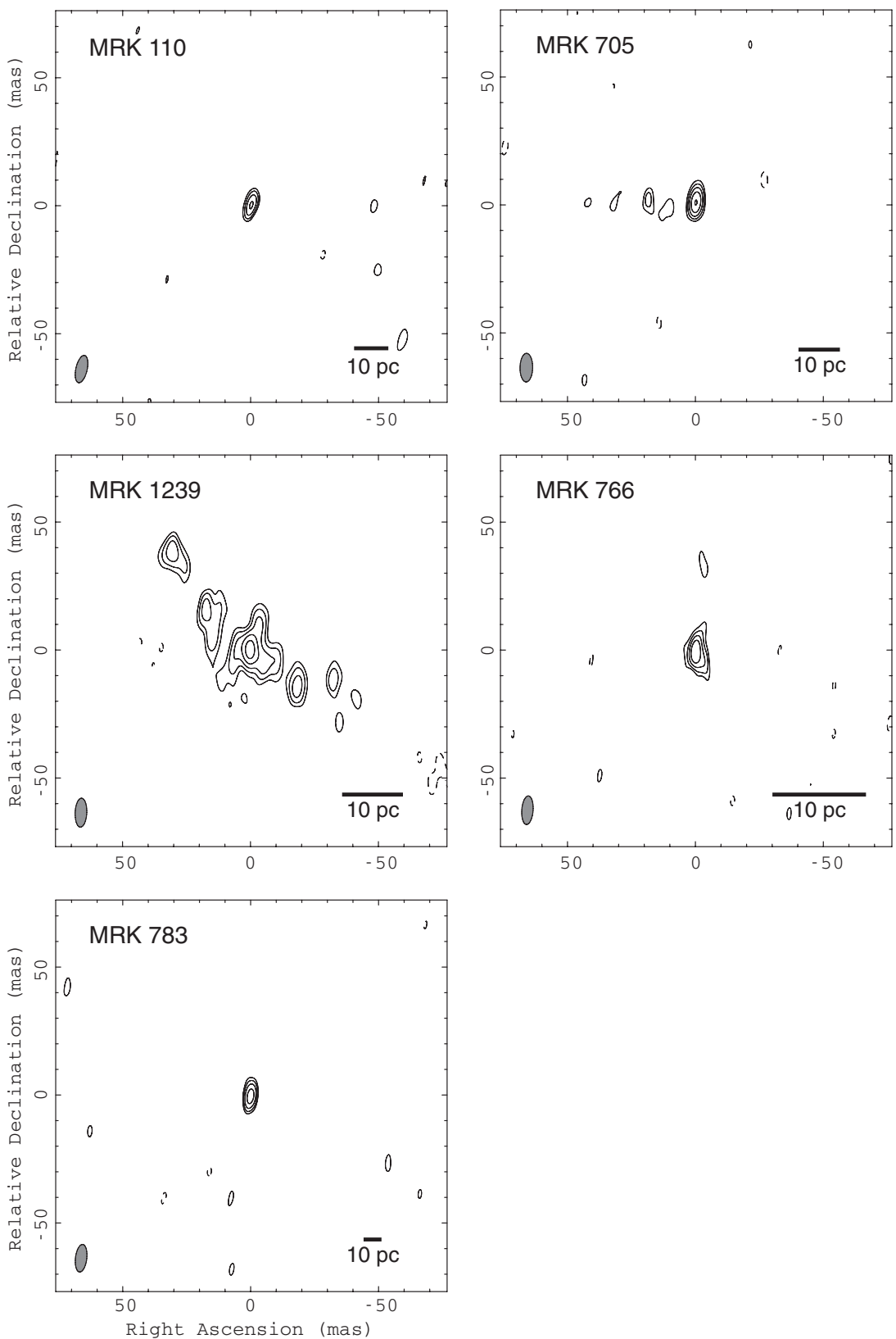

Figure 1. VLBA images with natural weighting for detected nearby radio-quiet NLS1s at $1.7 \mathrm{GHz}$ in the regions of $150 \times 150$ mas ${ }^{2}$. Contour levels are separated by factors of $\sqrt{2}$ beginning at three times the rms noise (Table 3). Negative and positive contours are represented by dashed and solid curves, respectively. Half-power beam sizes are given in the lower left corners. Angular scales corresponding to a linear scale of $10 \mathrm{pc}$ are given in the lower right corners.

found in a VLA archival image at sub-arcsec resolution (Figure 3(b) in the Appendix). MRK 705 showed a one-sided linear structure $\sim 45$ mas $(\sim 26 \mathrm{pc})$ east of the core. No larger structure has been found in VLA images (the Appendix). MRK 766 showed a signature of a northern elongation of $\sim 50$ mas $(\sim 13 \mathrm{pc})$ in the restored image (Figure 2$)$. The VLBA images for the other two sources (MRK 110 and MRK 783) exhibit apparently compact emission.

The VLBA-detected flux density was significantly lower than the VLA total flux density for all the sources, indicating low fractions of high-brightness components $\left(\gtrsim 5 \times 10^{6} \mathrm{~K}\right)$ relative to lower-brightness components (Section 4.1). In this paper, the radio core fraction, $f_{1.7 \mathrm{GHz}}^{10 \mathrm{pc}}$, is defined as the ratio of the flux density from the central $10 \mathrm{pc}$ (in diameter) to the total flux density at $1.7 \mathrm{GHz}$ in the rest frame. At the distances to our samples, $10 \mathrm{pc}$ typically corresponds to $\sim 20$ mas
(Column 10 in Table 3). Our VLBA baselines can retrieve $\geqslant 95 \%$ of the emissions as a correlated flux density at the minimum (projected) length of $\sim 210 \mathrm{~km}$ for the 20 mas radio size. Hence, the fluxes from the central $10 \mathrm{pc}$ were practically measurable (Column 11 in Table 3). On the other hand, we cannot rule out the possibility that emissions with much larger angular scales were partially or scarcely detected ("resolved out"). Hence, we adopted the total flux densities from the NRAO VLA Sky Survey (NVSS; Condon et al. 1998) at $1.4 \mathrm{GHz}$ as listed in Table 1; the contribution of the extended component was calculated by subtracting the $<10 \mathrm{pc}$ contribution at $1.4 \mathrm{GHz}$ from the NVSS value at the rest frame and then converted to the value at $1.7 \mathrm{GHz}$, assuming spectral indices of compact and extended components (see the Appendix and Table 3 in detail). Consequently, the core fractions were typically less than $10 \%$, up to $24 \%$ at most (Column 12 in Table 3). Because the entire radio structures are 

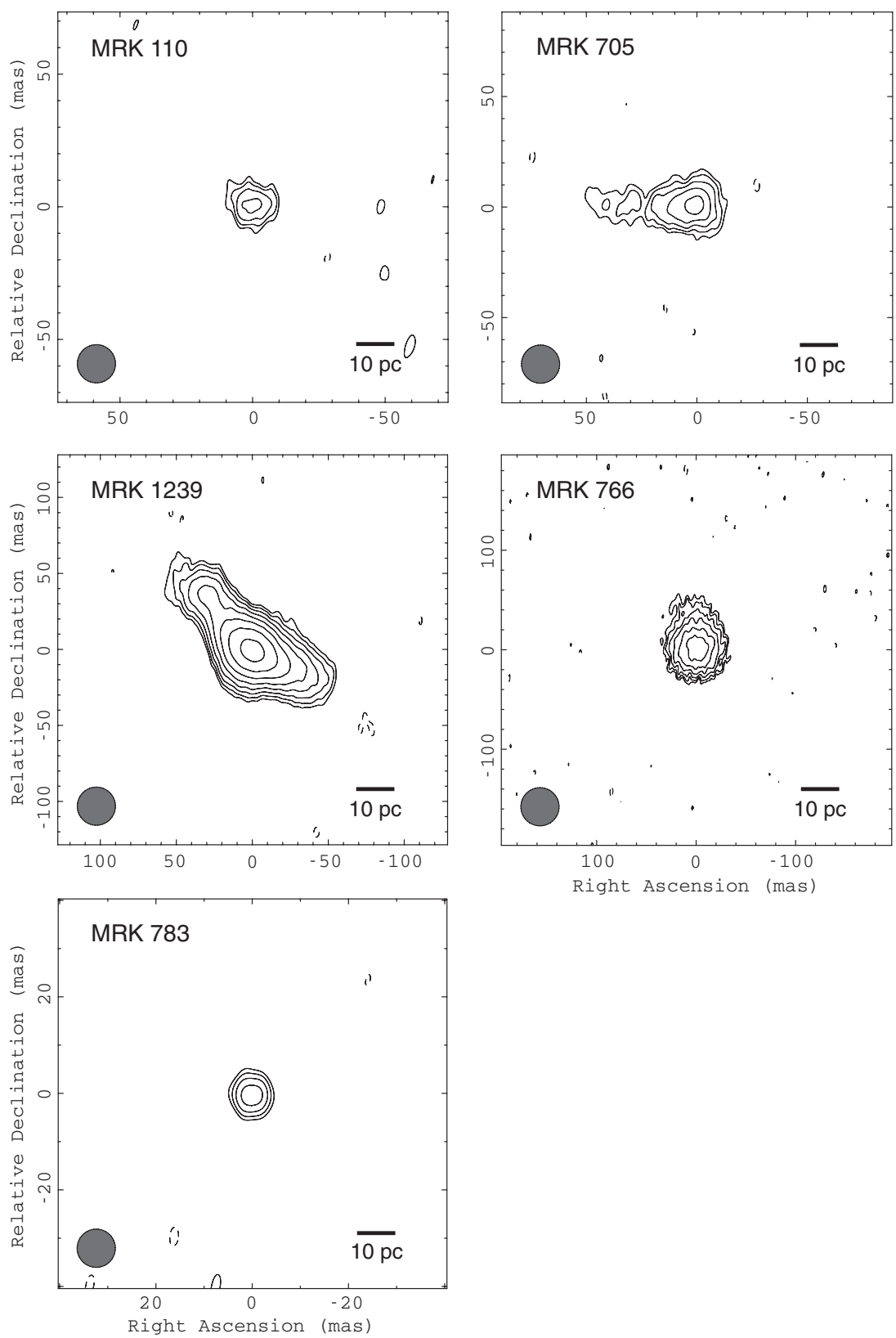

Figure 2. VLBA images restored with the $10 \mathrm{pc}$ beam in the regions of $205 \times 250 \mathrm{pc}^{2}$. Map descriptions are the same as for Figure 1 .

$\lesssim 300 \mathrm{pc}$ in the VLA images (Column 12 in Table 1), a significant portion of the emissions should originate in the regions of $\sim 10-300 \mathrm{pc}$ in the form of low-brightness components detected $\left(\gtrsim 5 \times 10^{6} \mathrm{~K}\right)$ and not detected $\left(\lesssim 5 \times 10^{6} \mathrm{~K}\right)$ in the VLBA images. In fact, we recognized a clear enhancement in the visibility amplitude of MRK 1239 at baselines of $\lesssim 3 \mathrm{M} \lambda$ (Figure 3(a) in the Appendix) and marginal enhancements also in MRK 766 and AKN 564 (the Appendix). These are evidences for the presence of a significant amount of emission components with sizes of $>70$ mas, corresponding to $>20-30$ pc for these sources.

\section{DISCUSSION}

\subsection{Compact Emission Components}

Our VLBA imaging provides the first insight into the pcscale region of several nearby radio-quiet NLS1s in a radio band. All five detected radio-quiet NLS1s have a compact radio component with a high-brightness temperature of $>6 \times 10^{7} \mathrm{~K}$. This is an evidence of a nonthermal process driven by jetproducing central engines in radio-quiet NLS1s, as well as radio-quiet Seyfert nuclei (e.g., Preuss \& Fosbury 1983; Neff $\&$ de Bruyn 1983) and radio-quiet quasars (Blundell \& Beasley 1998; Ulvestad et al. 2005).

It is notable that the central components make only minor contributions compared to the VLA total flux densities (i.e., low values of $f_{1.7 \mathrm{GHz}}^{10 \mathrm{pc}}$; Section 4.2). A previous study using the VLBA reported that all but 1 of 39 radio-selected lowluminosity AGNs were detected, and they exhibited coredominated structures in most cases (Nagar et al. 2005; see also Falcke et al. 2000; Anderson et al. 2004). However, the radio-quiet NLS1s in our sample present a contrasting aspect, although both of the AGN subclasses are weak radio sources in the nearby universe. The radio spectra are also quite different: flat or mildly inverted spectra in low-luminosity AGNs (Nagar 
et al. 2001; Anderson et al. 2004; Doi et al. 2005, 2011, 2013) but steep spectra in NLS1s (Moran 2000; Zhou \& Wang 2002). Such flat or inverted spectra would be accounted for by optically thick synchrotron emitters at the base of the jets (e.g., Falcke \& Biermann 1999). The scale-invariant jet model predicts that the radio luminosity from the jet bases depends on $\propto M_{\mathrm{BH}}^{17 / 12}$ in most accretion scenarios, where $M_{\mathrm{BH}}$ is the black hole mass (Heinz \& Sunyaev 2003). In fact, the known radio sources of low-luminosity AGNs tend to have large black holes of $M_{\mathrm{BH}} \sim 10^{8}-10^{9} M_{\odot}$ (e.g., Nagar et al. 2002a), in contrast to values of $\sim 10^{6}-10^{7} M_{\odot}$ for the radio-quiet NLS1s in our sample (Table 1) and the majority of nearby NLS1s (e.g., Doi et al. 2012; Grupe \& Mathur 2004; Zhou et al. 2006). Hence, compared with low-luminosity AGNs, the radio-quiet NLS1s possess less efficient radio cores.

Moreover, the radio-quiet NLS1s also exhibit significantly lower core contributions than radio-loud NLS1s (Doi et al. 2011); core enhancement due to the Doppler beaming effect is a natural explanation in some cases (Doi et al. 2007; Gu \& Chen 2010). In our VLBA images with limited dynamic ranges, we found no clear evidence of highly relativistic jets, such as unusually high brightness temperatures requiring Doppler boosting (Kellermann \& Pauliny-Toth 1969; Readhead 1994), for the radio-quiet NLS1s. We will present a comprehensive study of the core activity of both radio-quiet and radio-loud NLS1s in another paper (A. Doi et al., in preparation).

\subsection{Extended Emission Components}

Past VLA surveys of NLS1s found a combination of two radio properties: compact sources $(\lesssim 300 \mathrm{pc})$ and very steep spectra (Ulvestad et al. 1995; Moran 2000; Zhou \& Wang 2002). This had indicated that the radio sources should be localized but physically not so compact that they become optically thin in synchrotron radiation. Our VLBA observations have confirmed this picture at the pc scale.

As an example, MRK 1239 is only slightly resolved in VLA images at arcsecond resolutions and is revealed to exhibit very diffuse structures within $40 \mathrm{pc}$ in the VLBA image. Less than half of the total fluxes were detected in the VLBA image and originate in low-brightness components of $\sim 5 \times 10^{6} \mathrm{~K}$ (the Appendix). We examine the possibility of the thermal process (free-free emission) for radio-emitting plasma of $15.2 \mathrm{mJy}$ (the VLBA flux density of the extended component for MRK 1239). The brightness temperature of $5 \times 10^{6} \mathrm{~K}$ gives a physical temperature of $\leqslant 5 \times 10^{6} \mathrm{~K}$. According to the Gaunt factors of $\sim 10$ and $\sim 0.4$ at $\sim 1 \mathrm{GHz}$ (Gayet 1970) and $\sim 1 \mathrm{keV}$ (Sutherland 1998) for plasma at $\sim 10^{6} \mathrm{~K}$, the expected X-ray luminosity would be $>4 \times 10^{44} \mathrm{erg} \mathrm{s}^{-1}$ at $0.1-2.4 \mathrm{keV}$ (Rybicki \& Lightman 1979), much higher than $1 \times 10^{42} \mathrm{erg} \mathrm{s}^{-1}$ observed by ROSAT toward MRK 1239 (Zimmermann et al. 2001; see a similar discussion for radio-quiet quasars by Laor \& Behar 2008; Steenbrugge et al. 2011). It is difficult to reconcile a thermal process with the observed low-brightness radio emissions. The remaining lower-brightness components $\left(\lesssim 10^{6} \mathrm{~K}\right)$ that were below the VLBA sensitivity are presumably nonthermal jets as well because the position angle of the VLA-resolved structure of MRK 1239 (Figure 3(b) in the Appendix) is consistent with the direction of the two-sided jet-like structure in the VLBA image. A steep spectrum in total flux (Figure 3(c) in the Appendix) also strongly supports a nonthermal synchrotron origin for the diffuse emissions.

MRK 705 and MRK 766 also exhibit clear morphological evidences of jets in the VLBA images. A large portion of the total flux density comes from diffuse components $\left(f_{1.7 \mathrm{GHz}}^{10 \mathrm{pc}}=0.24\right.$ and 0.07, respectively), which is also the case for MRK 1239 $\left(f_{1.7 \mathrm{GHz}}^{10 \mathrm{pc}}=0.23\right)$. The other NLS1s also showed low core fractions $\left(f_{1.7 \mathrm{GHz}}^{10 \mathrm{pc}} \lesssim 0.10\right)$. NGC 4051 was not detected in our observation with $f_{1.7 \mathrm{GHz}}^{10 \mathrm{pc}}<0.05$ but was detected by Giroletti \& Panessa (2009) at $1.6 \mathrm{GHz}$ by very sensitive VLBI imaging. The radio morphology is symmetric and shows two-sided lobe-like emissions separated by $20 \mathrm{pc}$ and centered at a core of sub-mJy, which is aligned with larger-scale emissions in the VLA images (the Appendix). There is an additional radio-quiet NLS1 that was previously observed by VLBI. The radio core of NGC 5506, a hidden NLS1 candidate (Nagar et al. 2002b), is surrounded by several diffuse components in the pc-scale region; a bridging morphology indicates a physical connection between the core and the diffuse components via jets (Middelberg et al. 2004). Furthermore, a low-surface-brightness halo $\sim 350 \mathrm{pc}$ in diameter enshrouds the central features in a VLA image (Orienti \& Prieto 2010), and only one-third of the single-dish flux was detected by VLBI (Middelberg et al. 2004). Thus, a large portion of the radio luminosities come from diffuse emission components distributed within the nuclear regions $(\lesssim 300 p c)$, which is a general property of non-thermal jets in radio-quiet NLS1s.

These diffuse components are putative reservoirs of dissipated jet kinetic powers. For our samples $\left(\mathrm{M}_{\mathrm{BH}} \sim 10^{6}-10^{7} \mathrm{M}_{\odot}\right.$; Table 1) and the majority of nearby NLS1 populations $\left(\lesssim 10^{7} M_{\odot}\right.$; e.g., Zhou et al. 2006; Doi et al. 2012), the jet kinetic powers limited by the Eddington limit on the low-mass black holes may be insufficient to cause the jets to escape to kpc scales outside the host galaxies. Indeed, only a small number of known NLS1s exhibit kpc-scale radio morphology (Antón et al. 2008; Gliozzi et al. 2010; Doi et al. 2012); all of these NLS1s possess relatively high mass black holes of $\gtrsim 10^{7} M_{\odot}$ and estimated jet kinetic powers of $\gtrsim 10^{44} \mathrm{erg} \mathrm{s}^{-1}$ and show signatures of relativistic jets in most cases (Doi et al. 2012). On the other hand, even the most luminous radio source in our radio-quiet sample (MRK 705) possesses an extended radio power of $10^{23.5} \mathrm{~W} \mathrm{~Hz}^{-1}$ at $1.4 \mathrm{GHz}$, suggesting an estimated jet kinetic power of $10^{43.5} \mathrm{erg} \mathrm{s}^{-1}$ (by using the relation of O'Sullivan et al. 2011). These values are below the thresholds of the Fanaroff-Riley type I/II (Fanaroff \& Riley 1974) dichotomy at a radio power of $\sim 10^{24.6} \mathrm{~W} \mathrm{~Hz}^{-1}$ (Zamfir et al. 2008) and a kinetic power of $\sim 10^{44} \mathrm{erg} \mathrm{s}^{-1}$ (Punsly \& Zhang 2011) that enables escape in the form of supersonic radio lobes (Kawakatu et al. 2009; see the discussion in Doi et al. 2012 for NLS1s with kpc-scale radio structures). Furthermore, advancing radio lobes would be more likely to become disrupted by the dense interstellar medium in the nuclear region (e.g., De Young 1993) of spiral hosts, as seen in normal Seyfert galaxies (Orienti \& Prieto 2010). The diffuse components found in our NLS1 sample may also be relics of such disrupted structures. On the basis of the VLBI-observed radio-quiet and radio-loud NLS1s, we will present a study of their jet kinetic powers estimated from the extended radio components (A. Doi et al., in preparation).

\section{SUMMARY AND CONCLUSIONS}

Our VLBA imaging study at $1.7 \mathrm{GHz}$ and mas resolution provide the first insight into the pc-scale region of several nearby radio-quiet NLS1s in a radio band. Five of seven sources (MRK 110, MRK 705, MRK 1239, MRK 766, and MRK 783) were detected at brightness temperatures of $\gtrsim 5 \times 10^{6} \mathrm{~K}$ and contain radio cores with high-brightness temperatures of $>6 \times 10^{7} \mathrm{~K}$, indicating a nonthermal process driven by a jet- 
producing central engine, in common with other AGN classes. MRK 1239 exhibits a two-sided jet-like structure $\sim 40 \mathrm{pc}$ in extent. MRK 705 and MRK 766 exhibit one-sided linear jet structures of $\sim 26 \mathrm{pc}$ and $\sim 13 \mathrm{pc}$, respectively. On the other hand, two sources (NGC 4051 and AKN 564) were not detected at the sensitivity of our observations.

We defined the radio core fraction, $f_{1.7 \mathrm{GHz}}^{10 \mathrm{pc}}$, as the ratio of the flux density from the central $10 \mathrm{pc}$ (in diameter) to the total flux density at $1.7 \mathrm{GHz}$ in the rest frame. We found typical values of $f_{1.7 \mathrm{GHz}}^{10 \mathrm{Gc}}<0.1$ and a maximum of 0.24 in our sample. While radio sources are apparently compact at arcsec resolution $(\lesssim 300 \mathrm{pc})$, our VLBI study indicated that a large portion of the radio luminosities come from diffuse emission components that are distributed within the nuclear regions. Their central engines with low-mass black holes of $\sim 10^{6}-10^{7} M_{\odot}$ may supply jets with insufficient kinetic powers to escape to the outsides of the host galaxies.

The VLBA and VLA are operated by the NRAO, a facility of the National Science Foundation operated under cooperative agreement by Associated Universities, Inc. MERLIN is a National Facility operated by the University of Manchester at Jodrell Bank Observatory on behalf of STFC. We used NASA's Astrophysics Data System Abstract Service (ADS) and the NASA/IPAC Extragalactic Database (NED), which are operated by the Jet Propulsion Laboratory. This work was partially supported by a Grant-in-Aid for Scientific Research (C; 21540250 and B; 24340042, AD) from the Japan Society for the Promotion of Science (JSPS). This work was also partially supported by the Center for the Promotion of Integrated Sciences (CPIS) of Sokendai.

\section{APPENDIX}

\section{INDIVIDUAL RADIO-QUIET NLS1s}

MRK 110. Our VLBA image at $1.7 \mathrm{GHz}$ (Figure 1) showed a single component of $1.1 \mathrm{mJy}$, which retrieved $13 \%$ of the total flux density in the rest frame on the basis of the $1.4 \mathrm{GHz}$ NVSS flux density and a spectral index of $\alpha=-0.7$ between 1.4 and 8.4 GHz (Kukula et al. 1998). Because we could not make selfcalibration on this source owing to insufficient signal-to-noise ratios, we cannot rule out the possibility that the VLBA image was affected by some coherence loss in phase referencing. VLA A-array images showed a secondary component $33^{\prime \prime} 0(2.1 \mathrm{kpc})$ to the north of a central component (Kukula et al. 1998; see also Miller et al. 1993).

$M R K$ 705. The self-calibrated VLBA image of MRK 705 at 1.7 GHz showed a compact core of $1.8 \mathrm{mJy}$ and a possible linear structure that extends $\sim 45$ mas $(\sim 26 \mathrm{pc}$ ) to the east (Figures 1 and 2). An unresolved source of $8.5 \mathrm{mJy}$ was found in the FIRST image and accompanies a secondary source of $27.5 \mathrm{mJy}$, which has an unrelated infrared counterpart (Gallo et al. 2005). The VLA A-array image at $8.46 \mathrm{GHz}$ showed an unresolved $(<55 \mathrm{pc})$ source of $2.0 \mathrm{mJy}$ (Schmitt et al. 2001), which is consistent with the extent of the VLBA structure. A single component of $5.7 \mathrm{mJy}$ beam $^{-1}$ was seen at $1.7 \mathrm{GHz}$ in an archival image from the Multi-Element Radio Linked Interferometer Network (MERLIN) at 150 mas resolution (MERLIN proposal code: Y/32); a large portion of the MERLIN fluxes were not retrieved in the VLBA baselines. Because the emissions of MRK 705 were detected after self-calibration (Section 3.3), its astrometric position could not be obtained.
MRK 1239. MRK 1239 is an unresolved ( $<80 \mathrm{pc}$ ) source showing a steep spectrum in VLA A-array images (Ulvestad et al. 1995; Thean et al. 2000). However, our VLA A-array image from a good $u v$-coverage at $8.5 \mathrm{GHz}$ from the archival data AM384 showed a significantly elongated structure of $\sim 0^{\prime \prime} 12$, corresponding to $\sim 46 \mathrm{pc}$, at a position angle of $47^{\circ}$ (Figure $3(\mathrm{~b})$ ).

The self-calibrated VLBA image of MRK 1239 at $1.7 \mathrm{GHz}$ shows a low-brightness ( $2.4 \mathrm{mJy} \mathrm{beam}^{-1}$ or less) linear structure of $\sim 100$ mas $(\sim 40 \mathrm{pc})$ consisting of several components (Figure 1), which is consistent with the elongated size seen in the VLA image. The visibility amplitudes were clearly enhanced in baselines at $\lesssim 3 \mathrm{M} \lambda$ (Figure 3(a)). A visibility-based model fitting with a single-Gaussian source structure resulted in a major axis of 62 mas $(\sim 24 \mathrm{pc})$ at a position angle of $47^{\circ}$. The flux density of the Gaussian model was $21.5 \mathrm{mJy}$, consistent with the integrated flux density of $20.0 \mathrm{mJy}$ in the VLBA image (Table 3). Orienti \& Prieto (2010) also reported a VLBA image of MRK 1239 at $1.7 \mathrm{GHz}$ that showed two components of $10.2 \mathrm{mJy}$ in total aligned at a position angle similar to our result. The contribution from the central $10 \mathrm{pc}$ was $23 \%$ of the total flux density at the rest frame on the basis of the $1.4 \mathrm{GHz}$ NVSS flux density and assumed spectral indices of $\alpha=-0.5$ for the core and $\alpha=-0.54$ (below) for the extended components, respectively.

The presence of diffuse emission distributed within $\sim 50 \mathrm{pc}$ can also be inferred from our multi-frequency VLA B-array (quasi-simultaneous) observations (AD489; Figure 3(c)). A spectral index of $\alpha=-0.52$ was found at $1.4-5 \mathrm{GHz}$; steeper spectra appear at higher frequencies, $\alpha=-0.94$ at $5-8.5 \mathrm{GHz}$ and $\alpha=-1.64$ at $8.5-15 \mathrm{GHz}\left(S_{v} \propto v^{\alpha}\right)$. Such an apparent spectral break also appeared in the VLA A-array (not simultaneous) observations at $1.4-8.4 \mathrm{GHz}$ (not simultaneously) by Ulvestad et al. (1995). Moran (2000) pointed out that NLS1s tend to have significantly steeper radio spectra $(\alpha \sim 1.1-1.2)$ than classical Seyferts on the basis of VLA A-configuration observations at 1.4 and $8.4 \mathrm{GHz}$. They noted that the strong radiation field around highly accreting disks of NLS1s can steepen the synchrotron spectra owing to inverseCompton cooling of relativistic electrons in jets. However, our measurements for MRK 1239 from $u v$-tapered images of a common resolution resulted in a simple power-law spectrum with $\alpha=-0.54$. This provides evidence for resolution effects. The significantly steeper spectra generally seen in radio-quiet NLS1s might have been artificial, which may be related to the dominance of diffuse components (Section 5.2).

NGC 4051. NGC 4051 is one of two NLS1s not detected in our VLBA observation. To determine the phase-tracking center, we reanalyzed the VLA A-array $22 \mathrm{GHz}$ image of Hagiwara et al. (2003), which included nuclear $\mathrm{H}_{2} \mathrm{O}$ masers confined to a region of 0.'04; the VLA observation used the ICRF calibrator J1153+4931 as a reference position. Giroletti \& Panessa (2009) reported VLBI detection of NGC 4051 using the European VLBI Network (EVN) at both 1.6 and $5 \mathrm{GHz}$ at a position that differed by only $<10$ mas from our phase-tracking center (Table 2). Hence, it was unlikely that the nucleus of NGC 4051 was outside of the VLBA's FOV.

The EVN image at $1.7 \mathrm{GHz}$ showed a core $(0.45 \mathrm{mJy})$ and two symmetric sub-components $(0.73$ and $0.67 \mathrm{mJy})$ separated by $\sim 20$ pc with respect to the core with a maximum intensity of $\sim 0.34$ mJy beam $^{-1}$ (Giroletti \& Panessa 2009). Such weak emissions are consistent with our negative detection because they are $3 \sigma$ in the VLBA image. A MERLIN image at $1.6 \mathrm{GHz}$ showed a triple structure with an extent of 0.8 (38 pc) at 

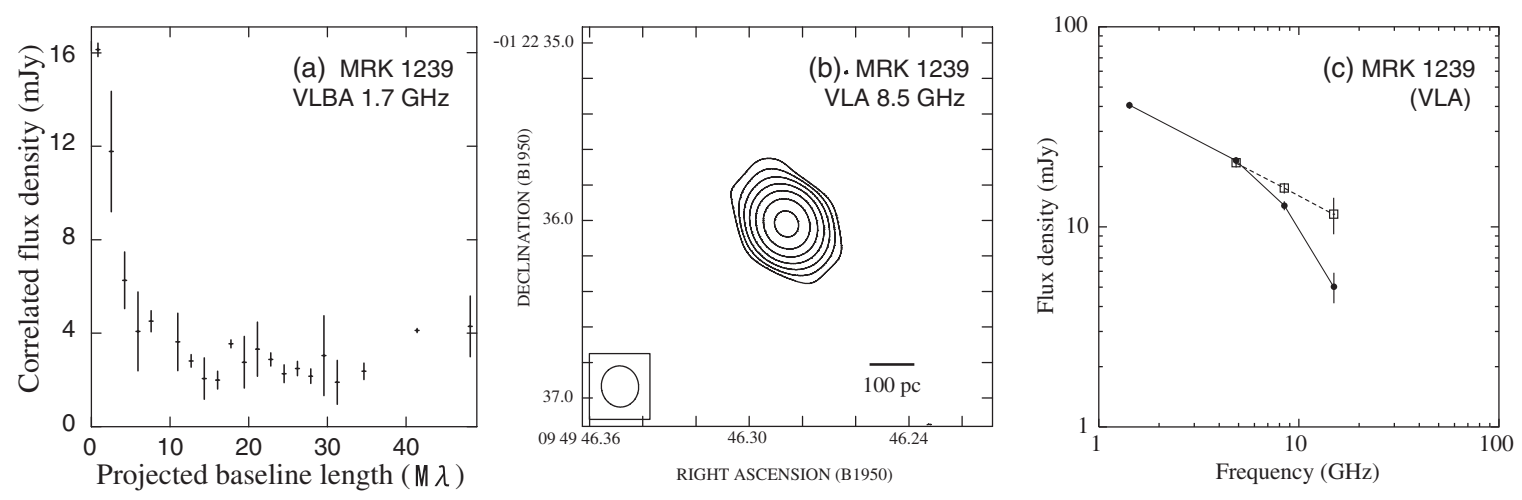

Figure 3. (a) Plot of visibility amplitude of MRK 1239 from VLBA data. Visibilities integrated every 60 minutes are divided into 30 bins over the range of the baseline lengths. (b) VLA images of MRK 1239 with VLA A-array at $8.5 \mathrm{GHz}$. Contour levels are $3 \sigma \times-1,1,2,4,8,16,32$, and 64 , where $\sigma=0.16 \mathrm{mJy}^{2}$ beam ${ }^{-1}$ is the image rms noise. The FWHM of the synthesized beam, $0 ! 27 \times 0.22$ at P.A. = 7.6, is illustrated in the lower left corner. (c) Radio spectra of MRK 1239 with VLA B-array. Filled circles connected with solid lines represent measurements from VLA images with uniform weighting. Open squares connected with dashed lines represent measurements from tapered images with a common beam size using the same data.

a position angle of $73^{\circ}$ (Christopoulou et al. 1997), which corresponds to the structure seen in the EVN image. The VLA A-array image at $1.4 \mathrm{GHz}$ showed larger structure extending up to $\sim 4^{\prime \prime}$ ( $\sim 330$ pc) from the nucleus (Ho \& Ulvestad 2001; Baum et al. 1993); the position angle has changed to $41^{\circ}$, perpendicular to the host-galaxy major axis $\left(135^{\circ}\right)$. The NVSS image shows very diffuse emission associated with the spiral arms and disk at a scale of arcmin, indicating stellar origins (Baum et al. 1993). Hence, we used a flux density of $14.7 \mathrm{mJy}$ in the VLA A-array image at $1.4 \mathrm{GHz}$ presented by Ho \& Ulvestad (2001) as the total flux density from an AGN contribution to derive the core fraction, assuming $\alpha=-0.7$ (Christopoulou et al. 1997).

$M R K$ 766. The self-calibrated VLBA image with natural weighting at $1.7 \mathrm{GHz}$ showed marginal structures extending to the southwest $\left(\right.$ P.A. $\sim-142^{\circ}$ ) and north (P.A. $\sim 0^{\circ}$ ) from the brightest component (Figure 1). In a restored image, diffuse emissions extending up to $\sim 50$ mas (corresponding to $\sim 13 \mathrm{pc}$ ) north were clearly seen (Figure 2 ). We recognized an enhancement in the visibility amplitude of MRK 766 at baselines of $<5 \mathrm{M} \lambda$; this suggests the presence of a significant amount of the diffuse component with the size of $>10 \mathrm{pc}$. Earlier VLA A-array images showed a slightly resolved structure $(\lesssim 100 \mathrm{pc})$ at a position angle of $\sim 30^{\circ}$ (Ulvestad et al. 1995; Nagar et al. 1999; Thean et al. 2000). A MERLIN image at $1.7 \mathrm{GHz}$ clearly showed a radio structure extending to $0.25(65 \mathrm{pc})$ at a position angle of $34^{\circ}$ (Thean et al. 2001), which is consistent with the southwest elongation in our VLBA image with natural weighting. The VLBA flux density was $2.5 \mathrm{mJy}$, which retrieved $7 \%$ of the total flux density at the rest frame on the basis of the $1.4 \mathrm{GHz}$ NVSS flux density and $\alpha=-0.72$ between 1.4 and $8.4 \mathrm{GHz}$ (Thean et al. 2001). Lal et al. (2004) reported a VLBI detection of MRK 766 at a relatively high intensity $7.1 \mathrm{mJy}^{\text {beam }}{ }^{-1}$ with a synthesized beam of $\sim 2$ mas at $5 \mathrm{GHz}$, which was inconsistent with a MERLIN archival image at $5 \mathrm{GHz}$ (MERLIN proposal code: 92NOVA) that showed diffuse emission of only $1.8 \mathrm{mJy}^{\text {beam }}{ }^{-1}$; this discrepancy might be because of variability. Because of the negative detection before self-calibration (Section 3.2), the astrometric position could not be obtained from our VLBA observation.

$M R K$ 783. Our VLBA image at $1.7 \mathrm{GHz}$ (Figure 1) showed a single component of $1.3 \mathrm{mJy}$, which retrieved $4 \%$ of the total flux density at the rest frame on the basis of the $1.4 \mathrm{GHz}$ NVSS flux density and assumed spectral indices of $\alpha=-0.5$ and -0.7 for the VLBA and diffuse components, respectively. The VLA
A-array image at $4.9 \mathrm{GHz}$ showed an unresolved ( $<380 \mathrm{pc})$ source of $3.5 \mathrm{mJy}$ (Ulvestad \& Wilson 1984).

$A K N$ 564. AKN 564 is one of two NLS1s not detected in our VLBA observation. The VLA A-array image at $8.46 \mathrm{GHz}$ showed a triple structure $(3.1,3.0$, and $0.9 \mathrm{mJy}$ ) extending $320 \mathrm{pc}$ to the north (Schmitt et al. 2001; Moran 2000). A MERLIN archival image showed somewhat diffuse emissions of only $1.04 \mathrm{mJy} \mathrm{beam}^{-1}$ at $5 \mathrm{GHz}$ with a 40 mas beam, which seemed to be consistent with our negative detection. On the other hand, a mas-scale structure with $3.7 \mathrm{mJy} \mathrm{beam}^{-1}$ had been detected at $5 \mathrm{GHz}$ by conventional fringe fitting (i.e., not phase referencing) using a much more sensitive VLBI array of $\sim 2$ mas resolution at $5 \mathrm{GHz}$ by Lal et al. (2004). However, they mentioned that they were not confident about the structure. The phase-tracking center of our VLBA observation was determined from the VLA archival data AB973 obtained with the A-array at $8.4 \mathrm{GHz}$ (Table 1) using the ICRF position of the phase calibrator $\mathrm{J} 2236+2828$ and differed by only $\sim 260$ mas from that of Lal et al. (2004). Hence, it was unlikely that the nucleus of AKN 564 was outside of the VLBA's FOV. Some amount of diffuse emissions could be in the VLBA FOV because the correlated amplitude was enhanced at baselines of $\lesssim 5 \mathrm{M} \lambda$, and the image noise was significantly higher than the expected thermal noise level (Section 3.3). Hence, we concluded that AKN 564 is dominated by diffuse components at pc scales, which are responsible for our negative detection. The spectral index between 1.4 and $4.9 \mathrm{GHz}$ is $\alpha=-0.74$ (Ulvestad et al. 1981).

\section{REFERENCES}

Abazajian, K. N., Adelman-McCarthy, J. K., Agüeros, M. A., et al. 2009, ApJS, 182,543

Abdo, A. A., Ackermann, M., Ajello, M., et al. 2009a, ApJ, 699, 976

Abdo, A. A., Ackermann, M., Ajello, M., et al. 2009b, ApJL, 707, L142

Anderson, J. M., Ulvestad, J. S., \& Ho, L. C. 2004, ApJ, 603, 42

Antón, S., Browne, I. W. A., \& Marchã, M. J. 2008, A\&A, 490, 583

Baum, S. A., O’Dea, C. P., Dallacassa, D., de Bruyn, A. G., \& Pedlar, A. 1993, ApJ, 419, 553

Beasley, A. J., \& Conway, J. E. 1995, in ASP Conf. Ser. 82, Very Long Baseline Interferometry and the VLBA, ed. J. A. Zensus, P. J. Diamond, \& P. J. Napier (San Francisco, CA: ASP), 328

Becker, R. H., White, R. L., \& Helfand, D. J. 1995, ApJ, 450, 559

Blandford, R. D., \& Konigl, A. 1979, ApJ, 232, 34

Blundell, K. M., \& Beasley, A. J. 1998, MNRAS, 299, 165

Boller, T., Brandt, W. N., \& Fink, H. 1996, A\&A, 305, 53

Boroson, T. A. 2005, AJ, 130, 381

Boroson, T. A., \& Green, R. F. 1992, ApJS, 80, 109 
Brandt, N., \& Boller, T. 1998, AN, 319, 7

Christopoulou, P. E., Holloway, A. J., Steffen, W., et al. 1997, MNRAS, 284,385

Condon, J. J., Cotton, W. D., Greisen, E. W., et al. 1998, AJ, 115, 1693

Dallacasa, D., Bondi, M., Alef, W., \& Mantovani, F. 1998, A\&AS, 129, 219

D’Ammando, F., Orienti, M., Finke, J., et al. 2012, MNRAS, 426, 317

De Young, D. S. 1993, ApJL, 405, L13

Doi, A., Asada, K., \& Nagai, H. 2011, ApJ, 738, 126

Doi, A., Fujisawa, K., Inoue, M., et al. 2007, PASJ, 59, 703

Doi, A., Kameno, S., Kohno, K., Nakanishi, K., \& Inoue, M. 2005, MNRAS, 363,692

Doi, A., Kohno, K., Nakanishi, K., et al. 2013, ApJ, in press (arXiv:1301.4757)

Doi, A., Nagai, H., Asada, K., et al. 2006, PASJ, 58, 829

Doi, A., Nagira, H., Kawakatu, N., et al. 2012, ApJ, 760, 41

Done, C., \& Gierliński, M. 2005, MNRAS, 364, 208

Dunlop, J. S., McLure, R. J., Kukula, M. J., et al. 2003, MNRAS, 340, 1095

Falcke, H., \& Biermann, P. L. 1999, A\&A, 342, 49

Falcke, H., Nagar, N. M., Wilson, A. S., \& Ulvestad, J. S. 2000, ApJ, 542,197

Fanaroff, B. L., \& Riley, J. M. 1974, MNRAS, 167, 31P

Fey, A. L., \& Charlot, P. 2000, ApJS, 128, 17

Foschini, L. 2011, in Proc. Conf. Narrow-Line Seyfert 1 Galaxies and Their Place in the Universe, ed. L. Foschini et al., published online at http://pos.sissa.it/cgi-bin/reader/conf.cgi?confid=126, 24

Gallo, L. C., Balestra, I., Costantini, E., et al. 2005, A\&A, 442, 909

Gayet, R. 1970, A\&A, 9, 312

Giroletti, M., \& Panessa, F. 2009, ApJL, 706, L260

Gliozzi, M., Papadakis, I. E., Grupe, D., et al. 2010, ApJ, 717, 1243

Goodrich, R. W. 1989, ApJ, 342, 224

Greene, J. E., Ho, L. C., \& Ulvestad, J. S. 2006, ApJ, 636, 56

Greisen, E. W. 2003, in Information Handling in Astronomy: Historical Vistas, ed. A. Heck (Astrophysics and Space Science Library, Vol. 285; Dordrecht: Kluwer), 109

Grupe, D., Komossa, S., Leighly, K. M., \& Page, K. L. 2010, ApJS, 187, 64

Grupe, D., \& Mathur, S. 2004, ApJL, 606, L41

Grupe, D., Wills, B. J., Leighly, K. M., \& Meusinger, H. 2004, AJ, 127, 156

Gu, M., \& Chen, Y. 2010, AJ, 139, 2612

Hagiwara, Y., Diamond, P. J., Miyoshi, M., Rovilos, E., \& Baan, W. 2003, MNRAS, 344, L53

Hayashida, K. 2000, NewAR, 44, 419

Heinz, S., \& Sunyaev, R. A. 2003, MNRAS, 343, L59

Ho, L. C. 2002, ApJ, 564, 120

Ho, L. C., \& Ulvestad, J. S. 2001, ApJS, 133, 77

Ivezić, Ž., Menou, K., Knapp, G. R., et al. 2002, AJ, 124, 2364

Kaspi, S., Smith, P. S., Netzer, H., et al. 2000, ApJ, 533, 631

Kawakatu, N., Kino, M., \& Nagai, H. 2009, ApJL, 697, L173

Kellermann, K. I., \& Pauliny-Toth, I. I. K. 1969, ApJL, 155, L71

Kellermann, K. I., Sramek, R., Schmidt, M., Shaffer, D. B., \& Green, R. 1989, AJ, 98, 1195

Komatsu, E., Dunkley, J., Nolta, M. R., et al. 2009, ApJS, 180, 330

Komossa, S., Voges, W., Xu, D., et al. 2006, AJ, 132, 531

Kovačević, J., Popović, L. Č., \& Dimitrijević, M. S. 2010, ApJS, 189, 15

Kovalev, Y. Y., Petrov, L., Fomalont, E. B., \& Gordon, D. 2007, AJ, 133, 1236

Kukula, M. J., Dunlop, J. S., Hughes, D. H., \& Rawlings, S. 1998, MNRAS, 297,366

Kukula, M. J., Pedlar, A., Baum, S. A., \& O'Dea, C. P. 1995, MNRAS, 276, 1262

Lacy, M., Laurent-Muehleisen, S. A., Ridgway, S. E., Becker, R. H., \& White, R. L. 2001, ApJL, 551, L17

Lal, D. V., Shastri, P., \& Gabuzda, D. C. 2004, A\&A, 425, 99

Laor, A. 2000, ApJL, 543, L111

Laor, A., \& Behar, E. 2008, MNRAS, 390, 847

Leighly, K. M. 1999a, ApJS, 125, 297

Leighly, K. M. 1999b, ApJS, 125, 317

Leighly, K. M., \& Moore, J. R. 2004, ApJ, 611, 107

Lister, M. L., Aller, H. D., Aller, M. F., et al. 2009, AJ, 137, 3718

Maccarone, T. J., Gallo, E., \& Fender, R. 2003, MNRAS, 345, L19

McLure, R. J., \& Jarvis, M. J. 2004, MNRAS, 353, L45

Merloni, A., Heinz, S., \& di Matteo, T. 2003, MNRAS, 345, 1057
Metcalf, R. B., \& Magliocchetti, M. 2006, MNRAS, 365, 101

Middelberg, E., Roy, A. L., Nagar, N. M., et al. 2004, A\&A, 417, 925

Miller, P., Rawlings, S., \& Saunders, R. 1993, MNRAS, 263, 425

Mineshige, S., Kawaguchi, T., Takeuchi, M., \& Hayashida, K. 2000, PASJ, 52,499

Moran, E. C. 2000, NewAR, 44, 527

Mullaney, J. R., \& Ward, M. J. 2008, MNRAS, 385, 53

Nagar, N. M., Falcke, H., \& Wilson, A. S. 2005, A\&A, 435, 521

Nagar, N. M., Falcke, H., Wilson, A. S., \& Ulvestad, J. S. 2002a, A\&A, 392, 53

Nagar, N. M., Oliva, E., Marconi, A., \& Maiolino, R. 2002b, A\&A, 391, L21

Nagar, N. M., Wilson, A. S., \& Falcke, H. 2001, ApJL, 559, L87

Nagar, N. M., Wilson, A. S., Mulchaey, J. S., \& Gallimore, J. F. 1999, ApJS, 120, 209

Neff, S. G., \& de Bruyn, A. G. 1983, A\&A, 128, 318

Orienti, M., \& Prieto, M. A. 2010, MNRAS, 401, 2599

Osterbrock, D. E. 1985, PASP, 97, 25

Osterbrock, D. E., \& Pogge, R. W. 1985, ApJ, 297, 166

O'Sullivan, E., Giacintucci, S., David, L. P., et al. 2011, ApJ, 735, 11

Peterson, B. M., McHardy, I. M., Wilkes, B. J., et al. 2000, ApJ, 542, 161

Pfefferkorn, F., Boller, T., \& Rafanelli, P. 2001, A\&A, 368, 797

Pounds, K. A., Done, C., \& Osborne, J. P. 1995, MNRAS, 277, L5

Preuss, E., \& Fosbury, R. A. E. 1983, MNRAS, 204, 783

Punsly, B., \& Zhang, S. 2011, ApJL, 735, L3

Readhead, A. C. S. 1994, ApJ, 426, 51

Ryan, C. J., De Robertis, M. M., Virani, S., Laor, A., \& Dawson, P. C. 2007, ApJ, 654,799

Rybicki, G. B., \& Lightman, A. P. 1979, Radiative Processes in Astrophysics (New York: Interscience), 393

Schmitt, H. R., Ulvestad, J. S., Antonucci, R. R. J., \& Kinney, A. L. 2001, ApJS, 132, 199

Shepherd, M. C. 1997, in ASP Conf. Ser. 125, Astronomical Data Analysis Software and Systems VI, ed. G. Hunt \& H. E. Payne (San Francisco, CA: ASP), 77

Steenbrugge, K. C., Jolley, E. J. D., Kuncic, Z., \& Blundell, K. M. 2011, MNRAS, 413, 1735

Stepanian, J. A., Benítez, E., Krongold, Y., et al. 2003, ApJ, 588, 746

Sulentic, J. W., Zwitter, T., Marziani, P., \& Dultzin-Hacyan, D. 2000, ApJL, 536, L5

Sutherland, R. S. 1998, MNRAS, 300, 321

Thean, A. H. C., Gillibrand, T. I., Pedlar, A., \& Kukula, M. J. 2001, MNRAS, 327,369

Thean, A. H. C., Pedlar, A., Kukula, M. J., Baum, S. A., \& O’Dea, C. P. 2000, MNRAS, 314, 573

Thompson, A. R., Moran, J. M., \& Swenson, G. W. 1986, Interferometry and Synthesis in Radio Astronomy (New York: Wiley)

Ulvestad, J. S., Antonucci, R. R. J., \& Barvainis, R. 2005, ApJ, 621, 123

Ulvestad, J. S., Antonucci, R. R. J., \& Goodrich, R. W. 1995, AJ, 109, 81

Ulvestad, J. S., \& Wilson, A. S. 1984, ApJ, 278, 544

Ulvestad, J. S., Wilson, A. S., \& Sramek, R. A. 1981, ApJ, 247, 419

Veron, P., Goncalves, A. C., \& Veron-Cetty, M.-P. 1997, A\&A, 319, 52

Véron-Cetty, M.-P., \& Véron, P. 2001, A\&A, 374, 92

Véron-Cetty, M.-P., Véron, P., \& Gonçalves, A. C. 2001, A\&A, 372, 730

Wang, T., Brinkmann, W., \& Bergeron, J. 1996, A\&A, 309, 81

Wang, T., \& Lu, Y. 2001, A\&A, 377, 52

Wrobel, J. M. 1995, in ASP Conf. Ser. 82, Very Long Baseline Interferometry and the VLBA, ed. J. A. Zensus, P. J. Diamond, \& P. J. Napier (San Francisco, CA: ASP), 411

Yuan, W., Zhou, H. Y., Komossa, S., et al. 2008, ApJ, 685, 801

Zamanov, R., Marziani, P., Sulentic, J. W., et al. 2002, ApJL, 576, L9

Zamfir, S., Sulentic, J. W., \& Marziani, P. 2008, MNRAS, 387, 856

Zhou, H., \& Wang, T. 2002, ChJAA, 2, 501

Zhou, H., Wang, T., Dong, X., Zhou, Y., \& Li, C. 2003, ApJ, 584, 147

Zhou, H., Wang, T., Yuan, W., et al. 2006, ApJS, 166, 128

Zhou, H., Wang, T., Yuan, W., et al. 2007, ApJL, 658, L13

Zimmermann, H.-U., Boller, T., Döbereiner, S., \& Pietsch, W. 2001, A\&A, 378,30

Zuther, J., Fischer, S., \& Eckart, A. 2011, in Proc. Conf. Narrow-Line Seyfert 1 Galaxies and Their Place in the Universe, ed. L. Foschini et al., published online at http://pos.sissa.it/cgi-bin/reader/conf.cgi?confid=126, 64 